\title{
A Distributed, Hybrid Energy-Efficient Clustering Protocol for Heterogeneous Wireless Sensor Network
}

\author{
Jun Wang ${ }^{1,2}$, Xuegang Zhu ${ }^{1}$,Yong Cheng ${ }^{2}$ and Yongsheng $\mathrm{Zhu}^{2}$ \\ ${ }^{1}$ Dept. of Computer \& Software, Nanjing University of Information Science \& \\ Technology, Nanjing 210044, China \\ ${ }^{2}$ Network Information Center, Nanjing University of Information Science \& \\ Technology, Nanjing 210044, China \\ wangjun@nuist.edu.cn, zhuxuegang2012@gmail.com
}

\begin{abstract}
The clustering Algorithm is a key technique used to prolong the lifetime of a sensor network by reducing energy consumption. It can prolong the network lifetime and improve scalability. In this paper, we propose a novel hybrid distributed energy-efficient heterogeneous clustered protocol for wireless sensor networks (HDEEHC). The HDEEHC protocol periodically selects cluster heads according to a hybrid of a primary parameter and a secondary parameter. The residual energy and the type of a node is the first parameter in the election of a cluster head, and the proximity to its neighbors or node degree is the second. The nodes which have high initial and residual energy will have more chances to be the cluster-heads than the low-energy nodes. The clustering does not depend on the network topology or size. Finally, the simulation results show that HDEEHC achieves a longer lifetime and more stability than HEED clustering protocols in heterogeneous environments.
\end{abstract}

Keywords: heterogeneous, Sensor networks, clustering, network lifetime, energy-efficiency

\section{Introduction}

With the advances in the technology of micro electro mechanical system (MEMS) and the great advancement in wireless communications, wireless sensor networks have emerged [1]. Recently, wireless sensor networks (WSNs) have become a one of the most popular network technology which has a potential of changing our lives drastically. It has become increasingly useful in all sorts of critical applications, such as battlefield surveillance, environmental monitoring [2], military application monitoring [3], intelligent office and traffic monitoring [4]. These various applications require high reliability of the sensor networks. In order to make sensor networks more reliable, the increasing attentions have been attracted to the researches on heterogeneous wireless sensor networks (HWSNs) in recent past [5-8]. Due to the small size, low cost, deployment flexibility and maintenance simplicity, sensor node can also be arranged in extremely poor condition, where battery replacement is difficult or even impossible to be performed. Therefore, a relevant scheme which has to develop to prolong the lifetime and increase the stability of the network by considering energy efficiency is essential for wireless sensor networks.

Network lifetime can be defined as the time elapsed until the first node in the network depletes its energy [9]. The clustering Algorithm is a key technique used to prolong the lifetime of a sensor network by reducing energy consumption [10]. Efficiently organizing sensor nodes into the different clusters is very useful in reducing 
energy consumption. Some energy-efficient routing protocols are designed based on the clustering structure like [9,11-14,16,17].

In this paper, we study the performance of clustering algorithms for heterogeneous wireless sensor networks in energy conservation. We assume that all nodes in the sensor network are equipped with different amount of energy level, which is a source of heterogeneity. It could be the result of re-energizing nodes or adding new nodes in order to extend the network lifetime [12]. Therefore, WSN is more likely to be heterogeneous networks rather than homogeneous ones. The protocols should be suitable for the characteristics of heterogeneous wireless sensor networks.

In this paper, we propose and evaluate a novel hybrid distributed energy-efficient heterogeneous clustered protocol for wireless sensor networks, which is called HDEEHC. Following the thoughts of HEED, HDEEHC considers a hybrid of energy and communication cost when selecting $\mathrm{CHs}$ and creates distributed clusters without the size and density of the sensor network being known. HDEEHC periodically selects cluster heads according to a hybrid of a primary parameter and a secondary parameter. The primary parameter is the residual energy of the nodes and the type of the nodes. The secondary parameter is node proximity to its neighbors or node degree which the same as HEED [9]. The nodes which have high initial and residual energy will have more chances to be the cluster-heads than the low-energy nodes. Therefore, HDEEHC can prolong the network lifetime, especially the stability period. Simulations show that HDEEHC achieves longer network lifetime and more stability than HEED clustering algorithms in heterogeneous environments.

The rest of the paper is organized as follows: In Section 2 we briefly review the heterogeneous cluster algorithm. In the 3rd section, we describe the heterogeneous WSN model. And then in the 4 section, we describe the whole structure of the algorithm. Section 5 shows the performance of HDEEHC by simulations and compares it with HEED. Finally, Section 6 gives concluding remarks.

\section{Related work}

The main aim of clustering scheme is to efficiently maintain the energy consumption of sensor nodes by involving them in multi-hop communication within a cluster and by performing data aggregation and fusion in order to decrease the number of transmitted messages to the sink and transmission distance of sensor nodes. There are two kinds of clustering algorithms. The clustering algorithms applied in homogeneous networks are called homogeneous clustering schemes, and the clustering algorithms applied in heterogeneous networks are referred to as heterogeneous ones.

At present there are some classical algorithms which are LEACH [11], HEED [9] and WLEACH [16]. Low Energy Adaptive Clustering Hierarchy (LEACH) [11] is a solution where clusters are dynamically created and well distributed to improve the energy load using probability. The LEACH algorithm selects cluster-heads periodically and drains energy uniformly by role rotation. In the homogeneous environments, LEACH performs very well, but its performance becomes badly in the heterogeneous network as shown by [12].

Unlike LEACH, Hybrid Energy-Efficient Distributed Clustering (HEED) [9] does not select cell-head nodes randomly. It is a distributed clustering scheme in which $\mathrm{CH}$ nodes are picked from the deployed sensors. HEED considers a hybrid of energy and communication cost when selecting CHs. Only sensors that have a high residual energy can become cell-head nodes. But under heterogeneous network, the low-energy nodes may own larger election probability than the high-energy nodes in HEED. So that the 
low-energy nodes will die more quickly than the high-energy ones, because HEED is unable to treat each node discriminatorily in term of the energy discrepancy.

Wise Low Energy Adaptive Clustering Hierarchy (WLEACH) [16] that is a improved of LEACH protocol by three improved sides: the first side is adding considering of energy ,the second aspect is adding multi-jump routing between nodes, and thethird aspect is adding dormancy of cluster head node.

Many heterogeneous clustering algorithms in various contexts have also been proposed in the past [12-14, 17], but to our knowledge, all of these algorithms are based on LEACH protocol and need to assume that the communication range of nodes must cover the entire network. So these algorithms are only suitable for small networks.

Stable Election Protocol (SEP) [12] was improved of LEACH protocol. It is among the first an energy efficiency routing protocol that used a two-level heterogeneous network, which is composed of two types of nodes according to the initial energy. In SEP algorithm, every sensor node independently elects itself as a cluster head based on its initial energy relative to that of other nodes.

Based on the SEP algorithm, Distributed energy efficient Clustering (DEEC) [13] algorithm is presented. In DEEC algorithm, cluster head is selected on the basis of probability of ratio of residual energy and average energy of the network. The nodes with high initial and residual energy have more opportunities than other nodes to become a cluster head node. Simulations show that DEEC achieves longer network lifetime and more effective messages than SEP algorithms in two-level heterogeneous environments.

Energy Efficient Heterogeneous Clustered (EEHC) [14], which is also based on LEACH, is a three-level heterogeneous WSNs. EEHC selects cluster heads based on the weighted probability of each node related to the initial energy, the more initial energy, the higher probability the node will be selected as a cluster head.

Improved and Balanced LEACH (IB-LEACH) [17] is a protocol that some high energy nodes elect themselves to be gateway at any given time with a certain probability and the non-gateway nodes elect themselves to be cluster heads with a certain probability. The non-cluster head determines to which cluster it wants to belong by choosing the cluster head that requires the minimum communication energy. Each cluster head collects and aggregates the data of their cluster members and transmits it to the chosen gateway which costs the minimum communication energy to reduce the energy consumption of cluster head and decreases probability of failure nodes.

\section{Heterogeneous network model}

In this section, we describe our system model of a wireless sensor network with nodes heterogeneous in their different initial amount of energy level. Firstly we describe the two-level heterogeneous networks and then consider the multi-level heterogeneous networks.

In the two-level heterogeneous networks, there are two types of sensor nodes, the normal nodes and advanced nodes. Suppose $E_{0}$ is the initial energy of each normal node, and $m$ is the fraction of the total number of nodes $N$, which are equipped with $a$ times more energy than the normal ones. Thus there are $m * N$ advanced nodes which own an initial energy of $(1+a) * E_{0}$, and $(1-m) * N$ normal nodes which own an initial energy of $E_{0}$.

For multi-level heterogeneous networks, initial energy of sensor nodes is randomly distributed over the close set $\left[E_{0}, E_{0}\left(1+a_{\max }\right)\right]$, where $E_{0}$ is the lower bound and $a_{\max }$ 
determines the value of the maximal energy. Initially, the node $s_{i}$ is equipped with an initial energy of $E_{0} *\left(1+a_{i}\right)$, which is $a_{i}$ times more energy than the lower bound $E_{0}$.

In addition to the above assumptions, we also assume that Nodes are quasi-stationary and location-aware which can be defined using GPS, signal strength or direction.

We do not make any assumptions about:

1) Network density or size

2) Nodes distribution;

3) The distribution of energy consumption among nodes;

4) The synchronization of the network.

\section{The HDEEHC Protocol}

In this section, we describe our HDEEHC protocol which is an extension of the HEED. HDEEHC protocol improves the stable region of the clustering hierarchy using the characteristic parameters of heterogeneity and is more effective in prolonging the network life time and the time interval before the death of the first node. The HDEEHC protocol periodically selects cluster heads according to a hybrid of a primary parameter and a secondary parameter. The residual energy and the type of a node is the first parameter in the election of a cluster head, and the proximity to its neighbors or node degree is the second similar to HEED. HDEEHC uses the residual energy and the type of the nodes to initialize the probability of nodes becoming a cluster head. HDEEHC algorithm for HWSNs has a periodic round; each round is divided into two different phases known as cluster formation and data communication.

\subsection{Cluster formation}

Similar to HEED protocol, the cluster formation of HDEEHC is divided into three different phases known as initialization phase, repetition phase and finalization phase. After the cluster formation phase, each node in the network becomes either a cluster head or a member of the cluster, so that the entire network becomes a hierarchical network.

\subsubsection{Initialization Phase}

The main idea is for the sensor nodes to compute its intra-cluster communication cost and set its probability of becoming a cluster head before a node starts executing HDEEHC. The goal is to let sensors with a high residual energy become cluster-head nodes.

The secondary clustering parameter, intra-cluster communication cost, is similar to HEED. Using Eq. (1) given in [9], each sensor node computes its intra-cluster communication cost,

$$
A M R P=\frac{\sum_{i=1}^{M} M i n P \omega r_{i}}{M}
$$

where $M i n P w r_{i}$ is the minimum power level required by a node $v_{i}$ communicating with a cluster head $\mathrm{u}, 1 \leq i \leq M, M$ is the number of nodes within the cluster range. 
For homogenous network liked HEED algorithm, every node sets its probability of becoming a cluster head, $\mathrm{CH}_{\text {prob }}$, as follows:

$$
C H_{\text {prob }}\left(s_{i}\right)=C_{\text {prob }} * \frac{E_{i_{-} \text {residual }}}{E_{i_{-} \max }}
$$

where $C_{\text {prob }}$ is only used to limit the initial cluster head announcements and has no direct impact on the final clusters, $E_{i_{-} \text {residual }}$ is the estimated current residual energy in the node and $E_{i_{\_} \max }$ is a reference maximum energy (corresponding to a fully charged battery), which is typically identical for all nodes. The $\mathrm{CH}_{\text {prob }}$ value of a node, however, is not allowed to fall below a certain threshold $p_{\min }($ e.g., 10_4), that is selected to be inversely proportional to $E_{i_{-} \max }$.

From Eq. (2), we can see that $\mathrm{CH}_{\text {prob }}$ is relevant to the ratio of the initial energy and the residual energy of nodes, $E_{i_{-} \text {residual }} / E_{i_{-} \max }$. In homogenous networks, all the nodes are equipped with the same initial energy, thus $\mathrm{CH}_{\text {prob }}$ is only relevant to the residual energy of nodes. But under heterogeneous network, it can't really reflect the level of residual energy of nodes that every node uses Eq. (2) to set its $\mathrm{CH}_{\text {prob }}$. Because the initial energy of nodes is different, which some nodes are equipped with more energy resources than the others. In this case, the low-energy nodes will die more quickly than the high-energy ones. In order to keep $\mathrm{CH}_{\text {prob }}$ being only relevant to the residual energy of nodes, we replace the reference value $C_{\text {prob }}$ with the weighted probabilities which is given in Eq. (3) for normal and advanced nodes.

$$
P_{a d v}=(1+a) \times C_{p r o b}, \quad P_{n o r}=C_{p r o b}
$$

Therefore, $C_{\text {prob }}$ is changed into

$$
C H_{\text {prob }}\left(s_{i}\right)= \begin{cases}P_{a d v} \times \frac{E_{i_{-} \text {residual }}}{E_{i_{-} \max }} & \text { if } s i \text { is the advanced node } \\ P_{\text {nor }} \times \frac{E_{i_{-} \text {residual }}}{E_{i_{-} \max }} & \text { if } s i \text { is the normal node }\end{cases}
$$

Using Eq. (4), we can make sure that $\mathrm{CH}_{\text {prob }}$ is only relevant to the residual energy of nodes. In HDEEHC algorithm, every node uses Eq. (4) to set its $\mathrm{CH}_{\text {prob }}$ in the initialization phase.

This model can be easily extended to multi-level heterogeneous networks. We use the weighted probability shown in Eq. (5).

$$
C H_{\text {prob }}\left(s_{i}\right)=\left(1+a_{i}\right) \times \frac{E_{i_{-} \text {residual }}}{E_{i_{-} \max }}
$$

From Eq. (4) and (5), the nodes with more energy will have a high $\mathrm{CH}_{\text {prob }}$ and more chances to be the cluster-heads than the nodes with less energy. Thus the energy of network is well distributed in the evolving process. 


\subsubsection{Repetition Phase}

This phase is also similar to HEED. During this phase, every sensor goes through several iterations until it finds the $\mathrm{CH}$ which it can transmit to with the least transmission power (cost). If it hears from no $\mathrm{CH}$ in the cluster radius, the sensor node elects itself to be a $\mathrm{CH}$ and sends an announcement message to its neighbors informing them about the change of its status. Finally, each sensor node doubles its $\mathrm{CH}_{\text {prob }}$ value and then goes to the next iteration of this phase. It stops executing this phase when its $\mathrm{CH}_{\text {prob }}$ has reached 1. Therefore, there are 2 types of cell head status that a sensor node could announce to its neighbors:

- Tentative_CH: The sensor becomes a tentative $\mathrm{CH}$ if its $\mathrm{CH}_{\text {prob }}$ is less than 1 . It can change its status to a regular node at a later iteration if it finds a lower cost $\mathrm{CH}$.

- Final_CH: The sensor permanently becomes a $\mathrm{CH}$ if its $\mathrm{CH}_{\text {prob }}$ has reached 1 .

\subsubsection{Finalization phase}

During this phase, each sensor would make a final decision on its status. It either picks the least cost $\mathrm{CH}$ or pronounces itself as $\mathrm{CH}$.

\subsection{Data communication}

After cluster formation was completed, all the alive nodes in for each cluster will periodically collect data and send it to its cluster head node. Consequently, the cluster head will collect all the data and send it to the sink node if it can communicate direct with the sink node or it's a neighbor cluster head node which is closer than it from the base station.

\section{Simulation}

In this section, we evaluate the performance of our protocol via Simulation. We simulated HDEEHC and HEED using parameters which are similar to those in [15]. The parameters are listed in Table 1 and we ignore the effect caused by signal collision and interference in the wireless channel. To validate the performance of HDEEHC, we simulate a heterogeneous wireless sensor network in a field with dimensions $1000 \mathrm{~m} *$ $1000 \mathrm{~m}$. The total number of the sensors nodes $N=300$. Simulations with 300 nodes are run for cluster ranges of 75,100,150,200, 250, 300 meters. The normal and advanced nodes are randomly distributed over the field. This means that the horizontal and vertical coordinates of each sensor are randomly selected between 0 and the maximum value of the dimension. The sink node is placed in the middle. Each result represents the average of 30 simulation runs with the same parameters.

In the analysis, HDEEHC protocol and HEED protocol had been analyzed using the same energy model as proposed by [15]. In the process of transmitting an 1-bit message over a distance $\mathrm{d}$, the energy expended by the radio is given by:

$$
E_{T x}(l, d)= \begin{cases}l E_{\text {elec }}+l \varepsilon_{f_{s}} d^{2}, & d<d_{0} \\ l E_{\text {elec }}+l \varepsilon_{\text {mp }} d^{2}, & d \geq d_{0}\end{cases}
$$

where $E_{\text {elec }}$ is the energy dissipated per bit to run the transmitter or the receiver circuit, and $\varepsilon_{\mathrm{fs}} \mathrm{d}^{2}$ or $\varepsilon_{\mathrm{mp}} \mathrm{d}^{4}$ is the amplifier energy that depend on the transmitter amplifier model. 
Table 1. Simulation parameters

\begin{tabular}{|c|c|c|}
\hline Type & Parameter & Value \\
\hline Network & $\begin{array}{l}\text { Network grid } \\
\text { Sink } \\
\mathrm{E}_{0}\end{array}$ & $\begin{array}{l}\text { From }(0,0) \text { to }(1000,1000) \\
\text { At }(500,500) \\
10 \mathrm{~J} / \text { battery }\end{array}$ \\
\hline Application & $\begin{array}{l}\text { Cluster radius } \\
\text { Data packet size } \\
\text { Broadcast packet size } \\
\text { Packet header size } \\
\text { Round (TNO) }\end{array}$ & $\begin{array}{l}75,100,150,200,250,300 \mathrm{~m} \\
100 \text { bytes } \\
25 \text { bytes } \\
25 \text { bytes } \\
5 \text { TDM frames }\end{array}$ \\
\hline Radio model & $\begin{array}{l}E_{\text {elec }} \\
\text { sfs } \\
\text { घmp } \\
E_{\text {fusion }} \\
\text { Threshold distance }\left(d_{0}\right)\end{array}$ & $\begin{array}{l}50 \mathrm{~nJ} / \mathrm{bit} \\
10 \mathrm{pJ} / \mathrm{bit} / \mathrm{m}^{2} \\
0.0013 \mathrm{pJ} / \mathrm{bit} / \mathrm{m}^{4} \\
5 \mathrm{~nJ} / \mathrm{bit} / \mathrm{signal} \\
75 \mathrm{~m}\end{array}$ \\
\hline
\end{tabular}

\subsection{Results under two-level heterogeneous networks}

We first observe the performance of HEED, and HDEEHC under two kinds of twolevel heterogeneous networks. Figure 1 shows the results of the case with $m=0.6$ and $a$ $=2$, and Figure 2 shows the results of the case with $m=0.4$ and $a=4$. It is obvious that the stable time and the lifetime of HDEEHC is prolonged compared to that of HEED. It is because that the advanced nodes which have high initial and residual energy will have more chances to be the cluster-heads than the low-energy nodes.

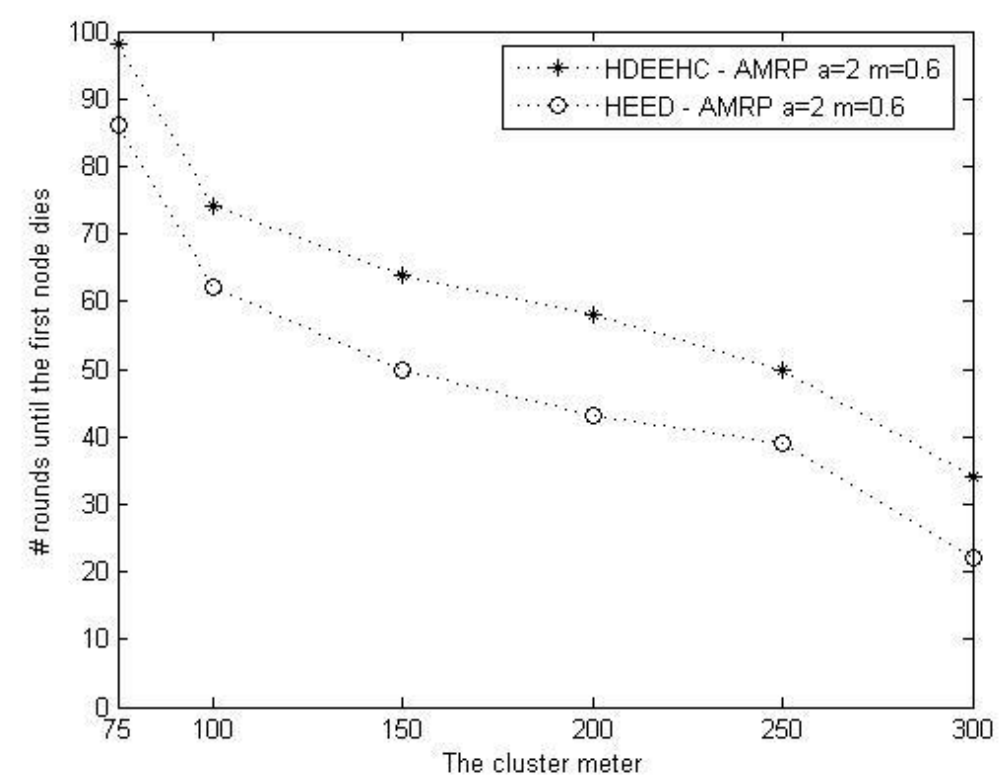

Figure 1. \# Rounds until the first node dies when $m=0.6$ and $a=2$ 


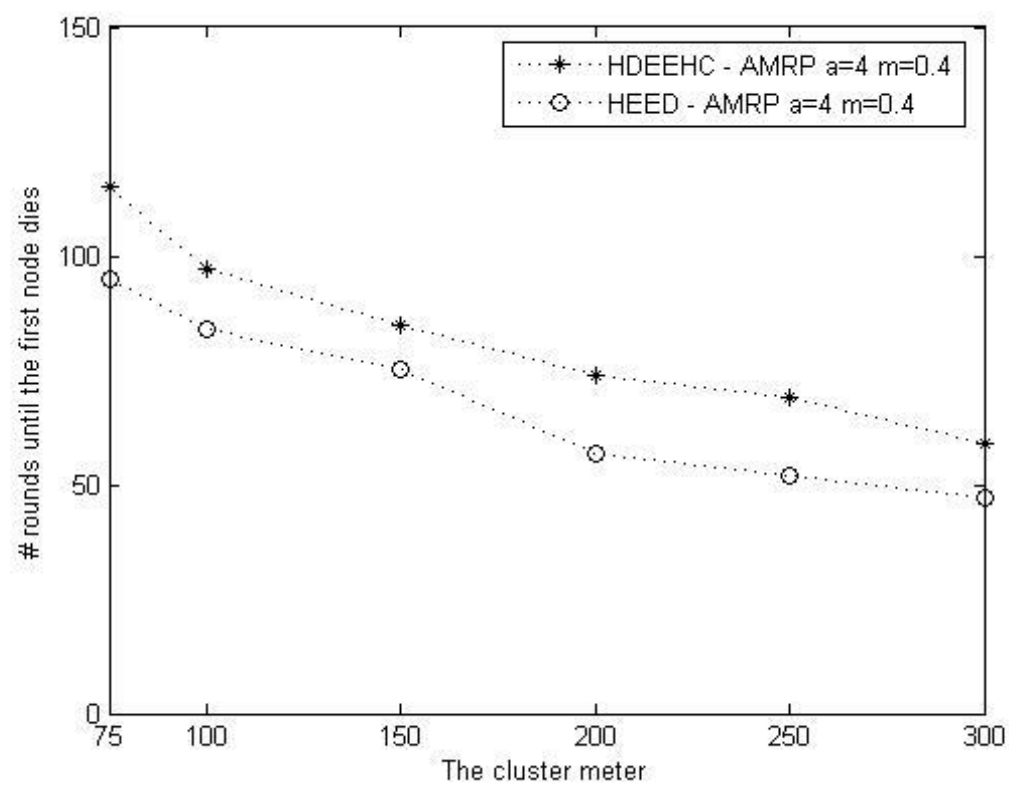

Figure 2. \# Rounds until the first node dies when $m=0.4$ and $a=4$

Then we increase the fraction $m$ of the advanced nodes from 0.1 to 0.9 . Figure 3 shows the number of rounds when the first node dies. We observe that HEED takes few advantages from the increase of total energy caused by increasing of $m$. The phenomenon mainly stems from the fact that the normal node haves lees chance to become a cluster head in HDEEHC.

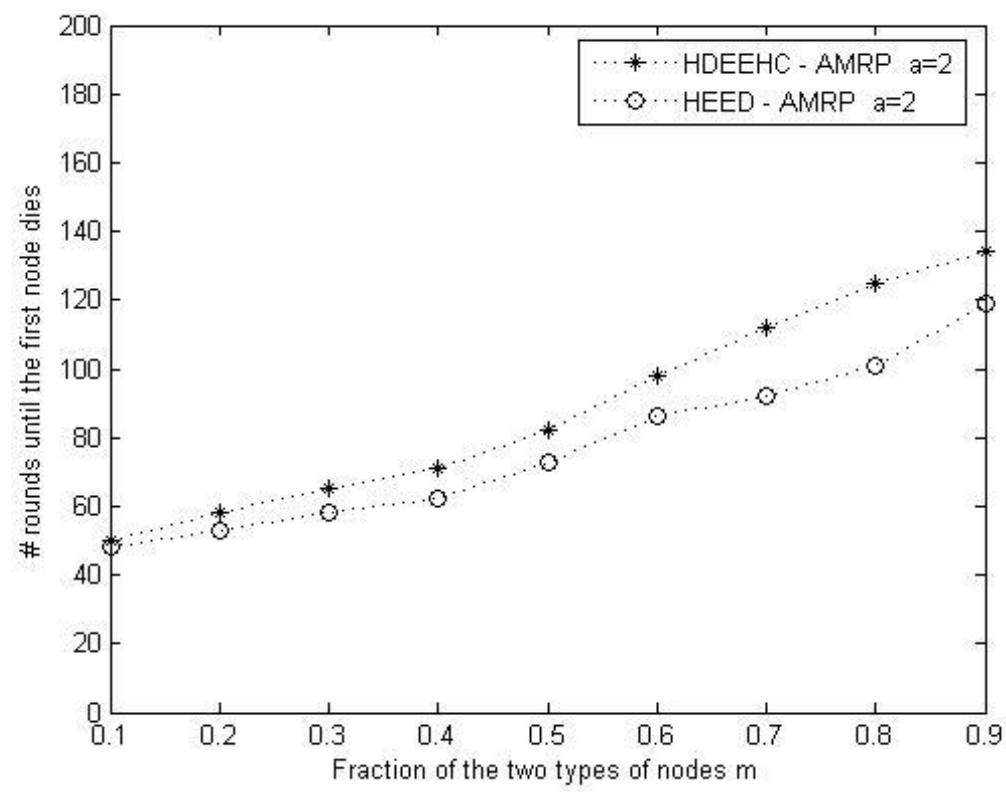

Figure 3. \# Rounds until the first node dies when $\boldsymbol{m}$ is varying and cluster radius is 75 


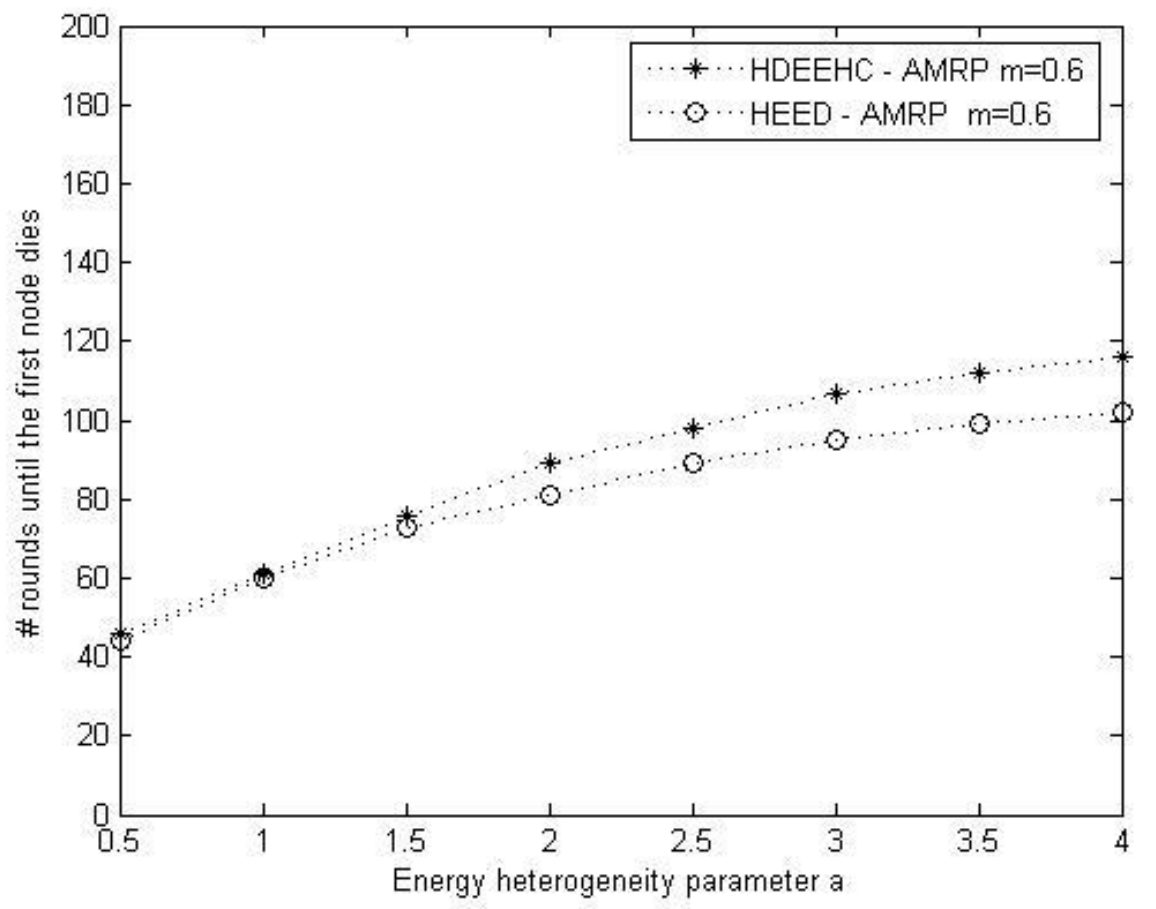

Figure 4. \# Rounds until the first node dies when $a$ is varying and cluster radius is 75

We increase the fraction $a$ of the advanced nodes from 0.5 to 4.0. Figure 4 shows the number of round when the first node dies. We observe that HEED also takes few advantages from the increase of total energy caused by increasing of $a$. This is because that the low-energy nodes may own larger election probability than the high-energy nodes in HEED.

\subsection{Results under multi-level heterogeneous networks}

For multi-level heterogeneous networks, the initial energy of nodes are randomly distributed in $\left[E_{0}, 4 E_{0}\right]$. To prevent the affection of random factors, the network is equipped with the same amount of initial energy. 


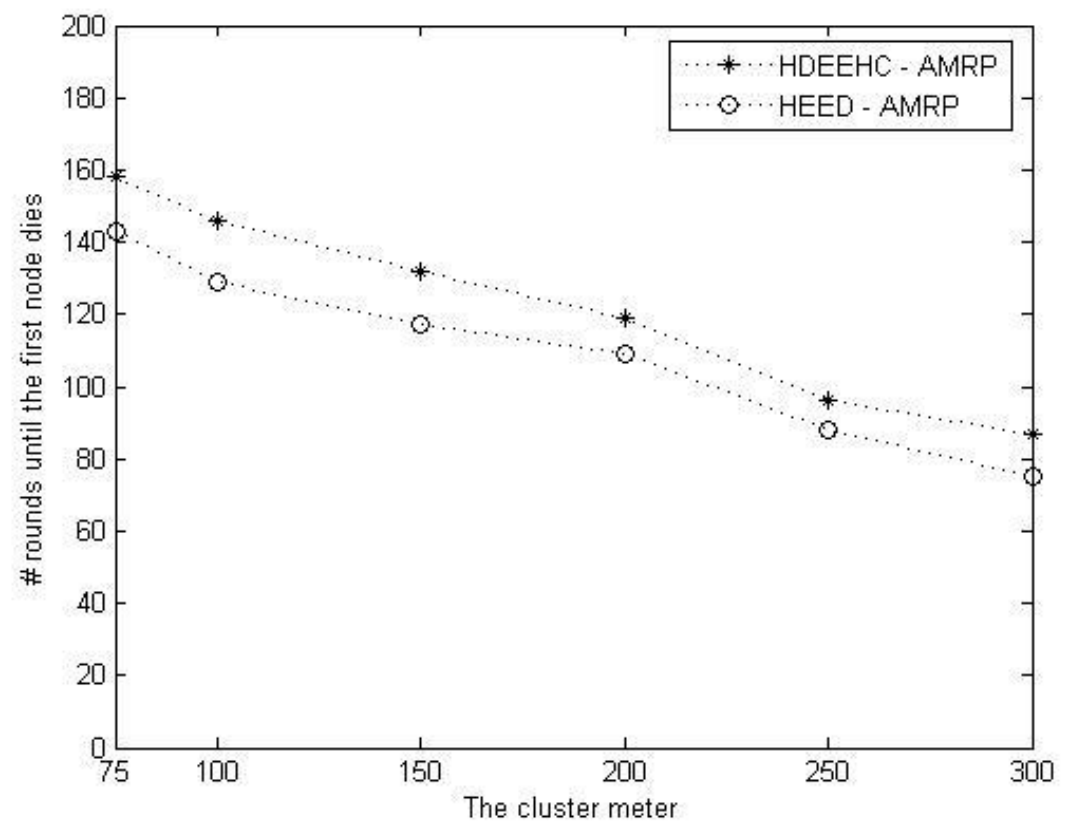

Figure 5. \#Rounds until the first node dies under multi-level heterogeneous networks

Figure 5 compares network lifetime with HDEEHC to HEED, where network lifetime is the time until the first node dies. We observe that HEED fails to take full advantage of the extra energy provided by the heterogeneous nodes. This is because that HEED treats all the nodes without discrimination. The results show that HDEEHC increases $15 \%$ more rounds of lifetime than HEED.

\section{Conclusions}

HDEEHC is an extension of the HEED, is more energy efficient and is more stability in prolonging the network life time using the characteristic parameters of heterogeneity in networks. Simulation results show that the HDEEHC achieves better performance in this respect, compared to HEED in heterogeneous environments.

\section{Acknowledgments}

The work in this paper has been supported by the National Natural Science Foundation of China (Grant No. 61005027), the Natural Science Foundation of the Jiangsu Higher Education Institutions of China (Grant No. 12KJB520009) and Scientific Research Fund of Nanjing University of Information \& technology (Grant No. 20110442).

\section{References}

[1] I. F. Akyildiz, W. Su, Y. Sankarasubramaniam and E. Cayirci, "Wireless sensor networks: a survey", Computer Networks, vol. 38, no. 4, (2002), pp. 393-422.

[2] J. K. Hart and K. Martinez, "Environmental Sensor Networks: A revolution in the earth system science", Earth-Science Reviews, vol. 78, (2006), pp. 177-191. 
[3] T. Bokareva, W. Hu, S. Kanhere, B. Ristic, N. Gordon, T. Bessell, M. Rutten and S. Jha, "Wireless sensor networks for battlefield surveillance", (2006), http://www.cse.unsw.edu.au/ tbokareva/papers/lwc.html.

[4] A. Hac, "Wireless Sensor Network Design", John Wiley and Sons, Ltd., (2003).

[5] E. J. Duarte-Melo and Liu Mingyan, "Analysis of energy consumption and lifetime of heterogeneous wireless sensor networks", Global Telecommunications Conference, GLOBECOM '02, IEEE, vol. 1, (2002) November, pp. 17-21.

[6] K. Lu, Y. Qian and J. Hu, "A framework for distributed key management schemes in heterogeneous wireless sensor networks", Performance, Computing, and Communications Conference, IPCCC 2006, 25th IEEE International, (2006) April.

[7] L. Yu, N. Wang, W. Zhang and C. Zheng, "Deploying a Heterogeneous Wireless Sensor Network", International Conference on Wireless Communications, Networking and Mobile Computing, WiCom, (2007) September 21-25, pp. 2588-2591.

[8] C. -H. Wu and Y. -C. Chung, "Heterogeneous Wireless Sensor Network Deployment and Topology Control Based on Irregular Sensor Model", Advances in Grid and Pervasive Computing Lecture Notes in Computer Science, vol. 4459/2007, (2007).

[9] O. Younis and S. Fahmy, "HEED: A Hybrid, Energy-Efficient Distributed Clustering Approach for Ad Hoc Sensor Networks", IEEE Transactions on Mobile Competing, vol. 3, no. 4, (2004), pp. 660-669.

[10] M. Younis, M. Youssef and K. Arisha, "Energyaware management in cluster-based sensor network", Computer Networks, vol. 43, no. 5, (2003), pp. 649-668.

[11] W. R. Heinzelman, A. P. Chandrakasan and H. Balakrishnan, "An application-specific protocol architecture for wireless micro sensor networks", IEEE Transactions on Wireless Communications, vol. 1, no. 4, (2002), pp. 660-670.

[12] G. Smaragdakis, I. Matta and A. Bestavros, "SEP: A Stable Election Protocol for clustered heterogeneous wireless sensor networks", Second International Workshop on Sensor and Actor Network Protocols and Applications (SANPA 2004), (2004).

[13]L. Qing, Q. Zhu and M. Wang, "Design of a distributed energy- efficient clustering algorithm for heterogeneous wireless sensor networks", ELSEVIER, Computer communications, vol. 29, (2006), pp. 2230- 2237.

[14]D. kumar, T. C. Aseri and R. B. Patel, "EEHC: Energy efficient heterogeneous clustered scheme for wireless sensor networks", Computer Communication, vol. 32, (2009), pp. 662-667.

[15] S. Bandyopadhyay and E. J. Coyle, "An energy efficient hierarchical clustering algorithm for wireless sensor networks", Proceeding of INFOCOM 2003, (2003) April.

[16] Y. Chen, Y. -l. Zhu and J. An, "An Energy-saving Routing Protocol Based on LEACH”, IEEE, (2012).

[17]L. I. Han, "An energy efficient routing algorithm for heterogeneous wireless sensor networks", International Conference on Computer Application and System Modeling (ICCASM), vol. 3, IEEE, (2010). 
International Journal of Grid and Distributed Computing Vol. 6, No. 4, August, 2013 